\title{
RIKEN NATURAL RADIOCARBON MEASUREMENTS VI
}

\section{FUMIO YAMASAKI, TATSUJI HAMADA, and CHIKAKO HAMADA}

Institute of Physical and Chemical Research (RIKEN)

Yamato-machi, Kita-Adachi-gun, Saitama Pref., Japan

The $\mathrm{C}^{14}$ dates given below are a continuation of the work presented in our previous list (Radiocarbon, 1969, v. 11, p. 451-462), and results obtained mainly during 1969 are described. A new $3.3 \mathrm{~L}$ copper counter was put into routine operation besides the $2.7 \mathrm{~L}$ stainless steel counter employed heretofore, yielding background counting rates of 8.5 and 5.5 cpm, respectively, when filled with dead $\mathrm{CO}_{2}$ at ca. $1.8 \mathrm{~atm}$.

Dates have been calculated on the basis of the $\mathrm{C}^{14}$ half-life of 5568 yr and $95 \%$ of NBS oxalic acid as modern standard.

\section{SAMPLE DESCRIPTIONS}

I. GEOLOGIC SAMPLES

\section{A. Japan}

\section{N-469. Esashi}

$$
910 \pm 110
$$

Peat from boggy flood plain of Horobetsu R., Kinkomanai, Esashicho, Esashi-gun, Hokkaido (45 $15^{\prime} \mathrm{N}$ Lat, $142^{\circ} 20^{\prime} \mathrm{E}$ Long), $120 \mathrm{~cm}$ below surface. Coll. and subm. 1969 by J. Nakamura, Kochi Univ.

\section{Furen series}

Material from boggy flood plain of Teshio R., Furen-cho, Kamikawagun, Hokkaido $\left(43^{\circ} 35^{\prime} \mathrm{N}\right.$ Lat, $142^{\circ} 10^{\prime} \mathrm{E}$ Long). Coll. and subm. 1969 by J. Nakamura. Comment: larger error is due to shortage of sample.

\section{N-470-1. Furen 1}

$1950 \pm 155$

Peat containing volcanic gravel from $40 \mathrm{~cm}$ below surface.

\section{N-470-2. Furen 2}

$9470 \pm 220$

Peat containing silt and sand from $100 \mathrm{~cm}$ below surface.

\section{Sotoyama series}

Material from terrace along Sotoyama R., Sotoyama, Morioka city, Iwate pref. (39 $43^{\prime} \mathrm{N}$ Lat, $141^{\circ} 2^{\prime} \mathrm{E}$ Long). Coll. and subm. 1969 by J. Nakamura.

\section{N-471-1. Sotoyama 1}

$1360 \pm 180$

Peat containing silt and sand from $70 \mathrm{~cm}$ below surface.

N-471-3. Sotoyama 3

Humic silt from $350 \mathrm{~cm}$ below surface. 
N-472. Kubokawa

$>\mathbf{3 7 , 8 0 0}$

Charred cone of Picea Polita from Yoshimi R. terrace at Kubokawacho, Takaoka-gun, Kochi pref. (33 $2^{\prime} \mathrm{N}$ Lat, $133^{\circ} 2^{\prime} \mathrm{E}$ Long). Coll. and subm. 1969 by J. Nakamura.

\section{N-598. Yamaji}

A.D. 1300

$650 \pm 105$

Piece of driftwood (Quercus glauca) from alluvium of Nakasuji R., Tosa-Nakamura city, Kochi pref. ( $32^{\circ} 54^{\prime} \mathrm{N}$ Lat, $133^{\circ} 0^{\prime} \mathrm{E}$ Long). Coll. 1968 and subm. by J. Nakamura.

\section{N-599. Toyonaga}

Piece of driftwood (Chamaecyparis sp.) from landslide debris along Yoshino R., $10 \mathrm{~m}$ below surface, Toyonaga, Otoyo-mura, Nagaoka-gun,

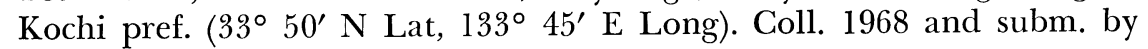
J. Nakamura.

\section{Yoshida-cho series}

Peat samples from boring core obtained at Yoshida-cho, Kochi city (33 $25^{\prime} \mathrm{N}$ Lat, $133^{\circ} 30^{\prime}$ E Long). Coll. 1968 and subm. by J. Nakamura (Nakamura, 1969).

N-600. Yoshida-cho 1

16.05 to $16.25 \mathrm{~m}$ below surface.

N-601. Yoshida-cho 2

19.20 to $20.00 \mathrm{~m}$ below surface.

N-602. Yoshida-cho 3

24.20 to $24.60 \mathrm{~m}$ below surface.

N-603. Yoshida-cho 4

29.50 to $30.10 \mathrm{~m}$ below surface. Comment (J.N.): pollen analysis indicates material is of Ice Age.

\section{Kaminoura series}

Peat from right bank of Kokai R., Kaminoura, Fujishiro-cho, Kitasoma-gun, Ibaraki pref. ( $35^{\circ} 53^{\prime} \mathrm{N}$ Lat, $140^{\circ} 8^{\prime} \mathrm{E}$ Long). Coll. 1968 by T. Sato; subm. by M. Oya, Waseda Univ. (Oya, 1969).

\section{N-610. Kaminoura 1}

$1750 \pm 110$

$67 \mathrm{~cm}$ below present surface of rice field.

$$
\text { A.D. } 200
$$

N-611. Kaminoura 2

$1140 \pm 100$

From just below N-610.

Peat from right bank of Kokai R., $80 \mathrm{~cm}$ below surface, Odome, 
Ryugasaki city, Kita-soma-gun, Ibaraki pref. (35 $54^{\prime} \mathrm{N}$ Lat, $140^{\circ} 9^{\prime} \mathrm{E}$ Long). Coll. 1968 by T. Sato; subm. by M. Oya.

\section{N-613. Ryugasakichobu}

$1410 \pm 100$

Peat from left bank of Tone R., $30 \mathrm{~cm}$ below surface, Ryugasakichobu, Kawachi-mura, Inashiki-gun, Ibaraki pref. (35 $52^{\circ} \mathrm{N}$ Lat, $140^{\circ}$ 13' E Long). Coll. 1968 by T. Sato; subm. by M. Oya.

\section{N-495. Kyu Nawa}

$17,900 \pm 400$

Wood from Kyu Nawa, Nawa-cho, Saihaku-gun, Tottori pref., $\mathrm{N}$ side of Mt. Daisen (35 $29^{\prime} \mathrm{N}$ Lat, $133^{\circ} 31^{\prime} \mathrm{E}$ Long), imbedded in thin clay layer overlain by mudflow containing pumice. Coll. 1966 and subm. by T. Kimachi, Yonago Kita High School. Comment (T.K.): wood ca. $1 \mathrm{~m}$ apart yielded $18,500 \pm 400$ (N-138, Radiocarbon, 1966, v. 8, p. 326).

\section{N-638. Ichihino}

$$
25,300 \pm 700
$$

Piece of charred timber, $15 \mathrm{~cm}$ diam., $50 \mathrm{~cm}$ long, from Ichihino, Hiwaki-cho, Satsuma-gun, Kagoshima pref. $\left(31^{\circ} 48^{\prime} \mathrm{N}\right.$ Lat, $130^{\circ} 25^{\prime}$ E Long), imbedded in Ito pyroclastic flow related to formation of Aira caldera. Coll. 1969 and subm. by S. Yokoyama, Toyko Univ. of Educ. Comment (S.Y.): other measurements on deposits are 16,350 \pm 350 (GaK-473, Radiocarbon, 1966, v. 8, p. 57; Aramaki, 1965) and 23,400 \pm 800 (GaK-558, Radiocarbon, 1966, v. 8, p. 57; Isshiki et al., 1965).

\section{N-618. Shitanohara}

Marine shell (Mya [Arenomya] arenaria) from Taito-misaki shell bed, Shitanohara, Misaki-cho, Isumi-gun, Chiba pref. (34 $57^{\prime} \mathrm{N}$ Lat, $139^{\circ}$ $50^{\prime}$ E Long). Coll. 1968 by S. Ohara, Chiba Univ.; subm. by K. Taira, Tokyo Univ. of Educ.

\section{N-609. Hayama}

A.D. 1065

$885 \pm 100$

Boat fragment from loam bed, ca. $3 \mathrm{~m}$ below surface, at Hayama, Hayama-cho, Kanagawa pref. (35 $20^{\prime} \mathrm{N}$ Lat, $139^{\circ} 36^{\prime} \mathrm{E}$ Long). Coll. 1967 by K. Watanabe, Tokyo Univ. of Educ.; subm. by K. Taira.

\section{Nansei Islands series}

Fossil hermatypic corals from various localities in Nansei Is. Coll. 1967-1968 and subm. by K. Konishi, Kanazawa Univ. Some samples dated also by ionium and protactinium method at Kanazawa Univ. (Omura, pers. commun.). Io ages are corrected for presence of initial ionium in sample (Omura, Konishi, and Hamada, 1969).

\section{N-545 a. Shirahama 1}


N.545 b. Shirahama 2

$$
4590 \pm 130
$$

2640 B.c.

$4490 \pm 130$

N-545 c. Shirahama 3

2540 B.C.

Coral heads 20 to $40 \mathrm{~cm}$ diam. in situ $6 \mathrm{~m}$ below low tide, present reef flat along Shirahama coast, ca. $500 \mathrm{~m} \mathrm{SW}$ of China, Okierabu-shima, Oshima-gun, Kagoshima pref. (27 $17^{\circ} \mathrm{N}$ Lat, $128^{\circ} 34^{\prime} \mathrm{E}$ Long). Io age: $1700 \pm 300,3100 \pm 300,1700 \pm 200 ; \mathrm{Pa}$ age: $7400 \pm 200,6600 \pm 200$, $5700 \pm 200$, respectively.

\section{N-546. Kasari 1}

$$
2130 \pm 115
$$

180 B.c.

Piece of coral head, ca. $30 \mathrm{~cm}$ diam. from bed hitherto mapped as Riukiu Limestone, ca. $1.5 \mathrm{~m}$ above low tide on Kasari coast, Amami-oshima, Oshima-gun, Kagoshima pref. (28 ${ }^{\circ} 2^{\prime} \mathrm{N}$ Lat, $129^{\circ} 42^{\prime} \mathrm{E}$ Long). Io age: $2000 \pm 300$; $\mathrm{Pa}$ age: 10,100 \pm 700 .

\section{N-547. Kasari 2}

Modern

Coral head, ca. $20 \mathrm{~cm}$ diam., ca. $1 \mathrm{~m}$ above low tide, from present reef flat, same location as N-546. Pa age: $69,000 \pm 3000$.

N-548. Tomori

$2270 \pm 115$

320 B.C.

Piece of coral head, ca. $20 \mathrm{~cm}$ diam., near pedestal surface of mushroom stack ca. $1.6 \mathrm{~m}$ above low tide, $400 \mathrm{~m}$ N of Tomori, Amami-oshima, Oshima-gun, Kagoshima pref. (28 $27^{\prime} \mathrm{N}$ Lat, $129^{\circ} 43^{\prime} \mathrm{E}$ Long).

\section{N-549. Sakamine 1}

$2330 \pm 110$

380 B.C.

Coral head, ca. $10 \mathrm{~cm}$ diam., in situ, ca. $1 \mathrm{~m}$ above high tide, from raised coral reef along Sakamine coast, Kikai-shima, Oshima-gun, Kagoshima pref. (28 $20^{\prime} \mathrm{N}$ Lat, $129^{\circ} 58^{\prime} \mathrm{E}$ Long).

\section{N-550. Sakamine 2}

$3810 \pm 120$

1860 B.c.

Coral head, ca. 30 to $40 \mathrm{~cm}$ diam. in situ, from raised coral reef, ca. $2 \mathrm{~m}$ above high tide, at rear of schoolyard of Sakamine Primary School, Nakaguma, Kikai-shima, Oshima-gun, Kagoshima pref. $\left(28^{\circ} 20^{\prime} \mathrm{N}\right.$ Lat, $129^{\circ} 58^{\prime} \mathrm{E}$ Long). Io age: $2900 \pm 150$; Pa age: $500 \pm 30$.

\section{N-551. Kunigami 1}

$3310 \pm 120$

1360 в.c.

Piece of coral head, ca. $30 \mathrm{~cm}$ diam. from emerged reef(?), ca. $2 \mathrm{~m}$ above high tide, at Kunigami-misaki, ca. $2.5 \mathrm{~km}$ ENE of Kunigami, Okierabushima, Oshima-gun, Kagoshima pref. $\left(27^{\circ} 25^{\prime} \mathrm{N}\right.$ Lat, $128^{\circ} 43^{\prime}$ E Long). Io age: $3600 \pm 200$; Pa age: $9400 \pm 400$.

\section{N-556. Kunigami 2}

Coral head, ca. $30 \mathrm{~cm}$ diam. in situ, from present reef flat, ca. 20 $\mathrm{cm}$ above low tide, at the same location as N-551. 
N-552. Kunigami 3

Coral head, ca. 40 to $50 \mathrm{~cm}$ diam. in situ, ca. $15 \mathrm{~m}$ above mean high tide, ca. $2.3 \mathrm{~km}$ ENE of Kunigami, Okierabu-shima, Oshima-gun, Kagoshima pref. (27 $26^{\prime} \mathrm{N}$ Lat, $128^{\circ} 43^{\prime} \mathrm{E}$ Long). Io age: $85,000 \pm 3000$; Pa age: $28,000 \pm 1000$.

\section{N-553. Kunigami 4}

Coral head, ca. $20 \mathrm{~cm}$ diam., ca. $20 \mathrm{~m}$ above mean high tide, 2.2 km ENE of Kunigami, Okierabu-shima, Oshima-gun, Kagoshima pref. (2726 $26^{\prime} \mathrm{N}$ Lat, $128^{\circ} 43^{\prime}$ E Long).

\section{N-554. Okidomari}

$3980 \pm 130$ 2030 B.c.

Coral head, ca. $20 \mathrm{~cm}$ in diam. in situ, from present reef flat, ca. 10 to $20 \mathrm{~cm}$ above low tide, at Okidomari coast, $900 \mathrm{~m}$ WSW of Shinjo, Okierabu-shima, Oshima-gun, Kagoshima pref. $\left(27^{\circ} 24^{\prime} \mathrm{N}\right.$ Lat, $128^{\circ} 34^{\prime}$ E Long).

\section{N-555. Wanjo-hama}

Coral head, ca. $20 \mathrm{~cm}$ in diam. in situ, from present reef flat, ca. $20 \mathrm{~cm}$ above low tide, at Wanjo-hama coast, $400 \mathrm{~m}$ NW of Azefu, Okierabushima, Oshima-gun, Kagoshima pref. (27 $24^{\prime}$ N Lat, $128^{\circ} 38^{\prime}$ E Long).

\section{B. Okinawa}

\section{Chinen series}

Coral from raised reef exposed at Chinen, $\mathrm{E}$ of Naha city, S Okinawashima $\left(26^{\circ} 30^{\prime} \mathrm{N}\right.$ Lat, $128^{\circ} 0^{\prime} \mathrm{E}$ Long). Coll. and subm. 1968 by K. Taira.

$$
\begin{aligned}
& \text { N-628. }+0.3 \mathrm{~m} \\
& \text { N-626. }+1 \mathrm{~m} \\
& \text { N-636. }+2 \mathrm{~m} \\
& \text { N-615. }+7 \mathrm{~m} \\
& \text { N-629. }
\end{aligned}
$$$$
4990 \pm 120
$$$$
3040 \text { B.c. }
$$$$
23,600 \pm 600
$$$$
21,650 \text { в.c. }
$$$$
25,700 \pm 800
$$$$
\text { 23,750 в.c. }
$$$$
37,300 \pm 2800
$$$$
\text { 34,350 B.c. }
$$$$
>37,800
$$$$
37,200 \pm 2900
$$$$
35,250 \text { в.C. }
$$$$
32,300 \pm 1700
$$$$
\text { 30,350 в.C. }
$$ 
N-635. $+40 \mathrm{~m}$

$30,800 \pm 1400$

28,850 B.c.

Comment: dates beyond 20,000 yr would be affected by modern carbon contamination.

\section{Mabuni series}

Coral from raised reef exposed at Mabuni, SSW of Naha city, S Okinawashima (26 $30^{\prime} \mathrm{N}$ Lat, $128^{\circ} 0^{\prime} \mathrm{E}$ Long). Coll. and subm. 1968 by K. Taira.

$$
\begin{aligned}
& \text { N-640. }+0.5 \mathrm{~m} \\
& \text { N-641. }+10 \mathrm{~m} \\
& \text { N-643. }+50 \mathrm{~m}
\end{aligned}
$$$$
32,500 \pm 1900
$$$$
\text { 30,550 в.c. }
$$

$36,600 \pm 2800$ 34,650 в.c.

\section{Taiwan}

Mainly fossil hermatypic corals from various localities in Taiwan. Coll. 1968 by T.-Y. H. Ma, Natl. Taiwan Univ., W. Hashimoto, and K. Taira; subm. by K. Taira. Comment (K.T.): dating of this series, as well as Okinawa and Borneo series (this list), is to establish sea-level curve during last $19,000 \mathrm{yr}$ in $\mathrm{E}$ Asia and to investigate problem of Jomon transgression which took place in Japan in 6000 yr в.P. but is inconsistent with data of Shepard (1964) and MacFarlan (1961).

\section{Lungkang series}

Wood from several horizons of Lungkang Formation at its type locality. Taken from wave-cut low cliff, $\mathrm{N}$ of Wumei-chi R., Houlung, Miaoli (24 $34^{\prime} \mathrm{N}$ Lat, $120^{\circ} 49^{\prime} \mathrm{E}$ Long). Other samples from same locality dated at Natl. Taiwan Univ. yielded $8415 \pm 433$ (NTU-2) and $6822 \pm$ 308 (NTU-3) (Hsu et al., 1965).

$$
\begin{aligned}
& \text { N-577. }+1 \mathrm{~m} \\
& \text { N-576. }+2 \mathrm{~m} \\
& \text { N-607. }+1.5 \mathrm{~m} \\
& \text { N-608. }+0.5 \mathrm{~m}
\end{aligned}
$$$$
7360 \pm 150
$$$$
5410 \text { в.c. }
$$$$
7180 \pm 140
$$$$
5230 \text { в.C. }
$$$$
7380 \pm 140
$$$$
5430 \text { B.c. }
$$

$7530 \pm 150$ 5580 в.c.

\section{Haikou series}

Reef corals from raised reef exposed at cut of natl. hwy. to Oluanpi $\mathrm{N}$ of Haikou, $\mathrm{N}$ of Hengchun, Pingtung-hsien $\left(22^{\circ} 1^{\prime} \mathrm{N}\right.$ Lat, $120^{\circ} 44^{\prime}$ E Long). Estimated $>20 \mathrm{~m}$ thick.

$$
\text { N.575. }+1 \text { m }
$$$$
1370 \pm 105
$$ 
N.606. $+5 \mathrm{~m}$

$5210 \pm 125$

N.605. +15 m

3260 B.c.

N-605. $+15 \mathrm{~m}$

$4050 \pm 115$

2100 B.c.

\section{Akungtien series}

Material from raised coral reef exposed at $\mathrm{S}$ foot of Mt. Hsiaokangshan, NE of Kangshan, Kaohsiung-hsien (22 $48^{\prime} \mathrm{N}$ Lat, $120^{\circ} 17^{\prime} \mathrm{E}$ Long). Outcrop of reef is ca. $4 \mathrm{~m}$ high, base is hidden by a corn field. Another sample from same locality dated at Natl. Taiwan Univ. yielded 7532 \pm 482 (NTU-4, Hsu et al., 1965).

\section{N-568. Akungtien 1}

$5560 \pm 105$

Coral from $0.6 \mathrm{~m}$ above field.

N-570. Akungtien 2

3610 B.c.

Coral from $0.8 \mathrm{~m}$ above field.

$5610 \pm 125$

3660 B.c.

N-569. Akungtien 3

$5510 \pm 125$

Coral from $1.9 \mathrm{~m}$ above field.

3560 в.C.

\section{N-574. Akungtien 4}

$5470 \pm 125$

Coral from $3.3 \mathrm{~m}$ above field.

\section{N-580. Akungtien 5}

Coral from $1.6 \mathrm{~m}$ above field.

3520 в.c.

\section{N-604. Akungtien 6}

$5370 \pm 125$

3420 B.c.

Marine shell (Ostrea sp.) from $4 \mathrm{~m}$ above field.

N-578. Akungtien 7

Coral from $3.0 \mathrm{~m}$ above field.

\section{N-614. Peinan}

Coral from raised reef exposed at coast, $\mathrm{N}$ of Peinan, NNW of Taitung (22 $48^{\prime} \mathrm{N}$ Lat, $121^{\circ} 10^{\prime} \mathrm{E}$ Long), at ca. $+100 \mathrm{~m}$.

\section{N-619. Chuanfanshih}

$32,600 \pm 1600$

\section{0,650 в.c.}

Coral from Chuanfanshih Limestone exposed along natl. hwy., $\mathrm{N}$ of Chuanfanshih, SE of Henchung, Pingtung-hsien $\left(22^{\circ} 1^{\prime} \mathrm{N}\right.$ Lat, $120^{\circ} 44^{\prime}$ E Long).

\section{K'enting series}

Material from raised coral reef ca. $7.5 \mathrm{~km} \mathrm{NE}$ of Henchung, Pingtung-hsien $\left(22^{\circ} 1^{\prime} \mathrm{N}\right.$ Lat, $120^{\circ} 44^{\prime} \mathrm{E}$ Long). 
N-620. K'enting 1

$4190 \pm 115$

2240 B.C.

Coral from $+12 \mathrm{~m}$ overlying silt, near K'enting Primary School.

N-621. K'enting 2

$7910 \pm 150$

5960 B.c.

Coral from $+20 \mathrm{~m}$, along natl. hwy., ca. $300 \mathrm{~m} \mathrm{NW}$ from N-620.

\section{N-627. K'enting 3}

$8420 \pm 150$

Coral from same level of and $5 \mathrm{~m}$ apart from N-621.

N-624. K'enting 4

6470 B.c.

Marine shell (Codakia sp.) from same locality as N-621.

\section{N-631. Wunchia}

$5550 \pm 125$

3600 B.c.

$7470 \pm 135$

5520 в.c.

Marine shell (Ostrea sp.) from Shikoshi Shell Beds of Tainan Formation exposed at $\mathrm{N}$ of Wunchia, ca. $15 \mathrm{~km}$ ESE of Tainan-shih, Tainanhsien $\left(23^{\circ} 0^{\prime} \mathrm{N}\right.$ Lat, $120^{\circ} 13^{\prime} \mathrm{E}$ Long).

\section{Chengkung series}

Coral from raised reef exposed at coast, $2.5 \mathrm{~km} \mathrm{~N}$ of Chengkung (23 $6^{\prime} \mathrm{N}$ Lat, $121^{\circ} 22^{\prime} \mathrm{E}$ Long).

N-616. $+15 \mathrm{~m}$

$25,900 \pm 800$

N-617. $+20 \mathrm{~m}$ 23,950 в.c.

N-642. Wangsha

$35,800 \pm 2400$

Coral from Wangsha Limestone exposed at $\mathrm{N}$ of Wangsha, $2 \mathrm{~km}$ of Hengchun, Pingtung-hsien (22。 $1^{\prime} \mathrm{N}$ Lat, $120^{\circ} 44^{\prime}$ E Long).

\section{N-645. Maashan}

$4570 \pm 120$

2620 B.C.

Piece of stock-like Bryozoa, ca. $10 \mathrm{~cm}$ diam., imbedded in Maashan Formation consisting of silt, exposed along road, at $\mathrm{N}$ of Maashan, ca. $4.5 \mathrm{~km} \mathrm{SSW}$ of Hengchun (22 ${ }^{\circ} 1^{\prime} \mathrm{N}$ Lat, $120^{\circ} 44^{\prime} \mathrm{E}$ Long). Comment (K.T.): date does not agree with expectation Lower Pleistocene or Upper Pliocene age.

\section{N-639. Hsiaochiang}

Coral from Fengpitou Limestone, Erhchiao Quarry, E of Hsiaochiang, ca. $15 \mathrm{~km} \mathrm{SE}$ of Kaohsiung, Kaohsiung-hsien $\left(22^{\circ} 38^{\prime} \mathrm{N}\right.$ Lat, $120^{\circ} 18^{\prime}$ E Long).

\section{N.644. Ssukou}

$20,600 \pm 400$

18,650 в.C.

Coral from emerged reef, Ssukou, ca. $2 \mathrm{~km}$ WNW of Hengchun, Pingtung-hsien (22。 $1^{\prime} \mathrm{N}$ Lat, $120^{\circ} 44^{\prime} \mathrm{E}$ Long). 


\section{Semporna series}

Coral from raised reef, E coast of Semporna, Semporna Peninsula, Borneo (4 $20^{\prime} \mathrm{N}$ Lat, $118^{\circ} 30^{\prime} \mathrm{E}$ Long). Coll. 1968 by W. Hashimoto; subm. by K. Taira.

\section{N-715. Semporna 1}

$0.5 \mathrm{~m}$ above high tide.

\section{N-716. Semporna 2}

$1.5 \mathrm{~m}$ above high tide.

\section{N-717. Semporna 3}

$1.5 \mathrm{~m}$ above high tide, and $2 \mathrm{~m}$ from N-716.

N-714. Semporna 4

$2 \mathrm{~m}$ above high tide. $\mathbf{3 5 , 0 0 0} \pm 2300$ 33,050 в.C.

$18,400 \pm 340$

16,450 в.c.

$29,200 \pm 1100$ 27,250 в.c.

$32,400 \pm 1600$ 30,450 в.c.

\section{E. Australia}

\section{Lake Keilambete series}

Organic and inorganic carbon from core and lakeshore outcrop of Quaternary lacustrine sediments in crater lake Keilambete, near Terang, Victoria, Australia ( $38^{\circ} 10^{\prime} \mathrm{S}$ Lat, $142^{\circ} 52^{\prime} \mathrm{E}$ Long). Lake water contains $903 \mathrm{ppm}$ of $\mathrm{CO}_{3}$, and core sediment contains up to $50 \%$ by weight of clay-sized carbonate; ostracod and Coxiella shells are present in core $4 \mathrm{~m}$ long from water $27 \mathrm{ft}$ deep. Coll. 1968 and subm. by J. M. Bowler, Australian Natl. Univ. Comment: materials treated by $\mathrm{HCl}$ and evolved $\mathrm{CO}_{2}$ dated as inorganic fraction. Dried residues are combusted in stream of oxygen and dated as organic fraction.

\section{N-388. Lake Keilambete LK2}

$3820 \pm 120$

Organic fraction of sandy peat containing Coxiella shells from bank of lake, $1 \mathrm{ft}, 7$ to $9 \mathrm{in}$. above 1967 water level and underlying youngest layer of indurated lake limestone.

\section{N-389. Lake Keilambete LK3}

$8690 \pm 165$

Organic fraction of sandy peat from bank of lake, 7 to 9 in. above 1967 water level underlying 2nd layer of indurated lake limestone.

\section{N-390. Lake Keilambete LK1}

$1890 \pm 115$

Piece of fallen tree lying half above, and half under water with indurated lake limestone and peaty mud over it. 
N-566. Lake Keilambete LK34

$$
29,100 \pm 1250
$$

27,150 в.c.

Inorganic carbon from lowest of 3 marl bands in shoreline volcanic sands, disconformably overlying Tertiary limestone. Sample provides limiting age of volcanic eruption and crater formation.

\section{N-567. Lake Keilambete LK37}

Inorganic carbon from highest of 3 marl bands assoc. with N-566.

N-517-1. Lake Keilambete LK4/11

Organic fraction, 11 to $21 \mathrm{~cm}$ in core.

N-517-2. Lake Keilambete LK4/11

Inorganic fraction of above sample.

N-518. Lake Keilambete LK4/21

Organic fraction, 21 to $33 \mathrm{~cm}$ in core.

N-519. Lake Keilambete LK4/55

Organic fraction, 55 to $65 \mathrm{~cm}$ in core.

N-520-1. Lake Keilambete LK4/79

Organic fraction, 79 to $90 \mathrm{~cm}$ in core.

N-520-2. Lake Keilambete LK4/79

Inorganic fraction of above sample.

N-521-1. Lake Keilambete LK4/102 Organic fraction, 102 to $112 \mathrm{~cm}$ in core.

N-521-2. Lake Keilambete LK4/102 Inorganic fraction of above sample.

N-522-1. Lake Keilambete LK4/130 Organic fraction, 130 to $140 \mathrm{~cm}$ in core.

N-522-2. Lake Keilambete LK4/130 Inorganic fraction of above sample.

N-523-1. Lake Keilambete LK4/165 Organic fraction, 165 to $175 \mathrm{~cm}$ in core.

N-523-2. Lake Keilambete LK4/165 Inorganic fraction of above sample.
A.D. 1340

$610 \pm 110$

$360 \pm 105$ A.D. 1590

$935 \pm 110$

A.D. 1015

$1970 \pm 115$

20 B.C.

$2410 \pm 120$
460 B.C.

$2560 \pm 120$

610 B.C.

$2600 \pm 110$

650 B.c.

$2900 \pm 120$

950 B.C.

$2970 \pm 120$

1020 B.c.

$4150 \pm 190$ 2200 B.C.

$3580 \pm 125$

1630 B.C.

$5430 \pm 135$

3480 B.C. 
N-524-1. Lake Keilambete LK4/190

$4200 \pm 125$

Organic fraction, 190 to $200 \mathrm{~cm}$ in core.

2250 в.c.

N-524-2. Lake Keilambete LK4/190

$5960 \pm 140$

4010 в.c.

Inorganic fraction of above sample.

N-525-1. Lake Keilambete LK4/235

$5250 \pm 135$

Organic fraction, 235 to $245 \mathrm{~cm}$ in core.

3300 B.c.

N-525-2. Lake Keilambete LK4/235

$5680 \pm 160$

Inorganic fraction of above sample.

N-526-1. Lake Keilambete LK4/290

Organic fraction, 290 to $300 \mathrm{~cm}$ in core.

N-526-2. Lake Keilambete LK4/290 3730 в.c.

$6440 \pm 145$ 4490 в.c.

Inorganic fraction of above sample.

N-527-1. Lake Keilambete LK4/325

$6290 \pm 140$

4340 в.c.

Organic fraction, 325 to $345 \mathrm{~cm}$ in core.

N-527-2. Lake Keilambete LK4/325

Inorganic fraction of above sample.

\section{N-528. Lake Keilambete LK4/395}

$7850 \pm 165$

5900 в.c.

$9860 \pm 180$

7910 B.c.

Organic fraction, 395 to $412 \mathrm{~cm}$ in core, containing trace of inorganic carbon, just below major disconformity. Comment (J.M.B.): this series forms part of a cooperative project in which Bowler is studying the Quaternary variations in lake levels believed to have been controlled by changes in climate. This sequence, back to 29,000 B.P. is the 1st from a lake core in Australia extending beyond the last glacial maximum.

\section{Pooraka series}

Samples from S bank of Dry Creek, Pooraka, S Australia $\left(34^{\circ} 50^{\prime}\right.$ S Lat, $138^{\circ}$ 37' E Long). Coll. 1969 and subm. by G. E. Williams, Univ. of Adelaide.

\section{N-633. Pooraka 1}

$34,600 \pm 2600$ 32,650 B.c.

Charcoal fragments and carbonized wood from late Pleistocene alluvial sand $3 \mathrm{~m}$ below top of bank.

\section{N-634. Pooraka 2}

$18,900 \pm 450$

Dense nodules of calcium carbonate from calcareous soil developed within late Pleistocene alluvium, $1 \mathrm{~m}$ below top of bank and directly above N-633. 
General Comment (G.E.W.): N-633 indicates Wisconsin age for last major episode of alluvial fan building in Adelaide region of $\mathrm{S}$ Australia (Williams, 1970). N-634 is consistent with stratigraphy, and suggestive of late Wisconsin for calcareous soil development.

\section{PEDOLOGIC SAMPLES}

Total organic carbon of humic horizon in volcanic ash soil from various localities coll. 1969 by Y. Yamada, Natl. Inst. Agric. Sci., and dated to determine relationship between soil age and properties of humus in soil.

N-669. Kamifuno Kfn-2

$5630 \pm 120$

Sample from $\mathrm{A}_{12}$ horizon, 8 to $21 \mathrm{~cm}$ below surface, at Kamifuno, Funo-mura, Futami-gun, Hiroshima pref. $\left(34^{\circ} 53^{\prime} \mathrm{N}\right.$ Lat, $132^{\circ} 47^{\prime} \mathrm{E}$ Long).

\section{Yokodani series}

Samples from Yokodani, Funo-mura, Futami-gun, Hiroshima pref. (34 $56^{\prime}$ N Lat, $132^{\circ} 44^{\prime}$ E Long).

\section{N-670. Yokodani 1 Ykd-1}

$5090 \pm 130$

From $\mathrm{A}_{13}$ horizon, 90 to $100 \mathrm{~cm}$ below surface.

\section{N-671. Yokodani 2 Ykd-2}

$6070 \pm 155$

4120 B.c.

From $A_{3}$ horizon, 100 to $110 \mathrm{~cm}$ below surface, just below N-670.

\section{Ozota series}

Samples from Ozota, Toyosaka-cho, Kamo-gun, Hiroshima pref. $\left(34^{\circ}\right.$ $37^{\prime} \mathrm{N}$ Lat, $132^{\circ} 48^{\prime} \mathrm{E}$ Long).

\section{N-672. Ozota 1 Ozt-2}

$1950 \pm 100$

From $A_{12}$ horizon, 18 to $33 \mathrm{~cm}$ below surface.

N.673. Ozota 2 Ozt-3

A.D. 0

$3580 \pm 130$

1630 B.c.

From $A_{13}$ horizon, 33 to $65 \mathrm{~cm}$ below surface, just below $\mathrm{N}-673$.

N-674. Hirodomeno

$970 \pm 110$

Sample from $\mathrm{A}_{11}$ horizon, 0 to $30 \mathrm{~cm}$ below surface, at Hirodomeno, Wakasa-cho, Yazu-gun, Tottori pref. (35 $24^{\prime}$ N Lat, 134 $27^{\prime}$ E Long).

\section{Nashibara series}

Samples from Nashibara, Saji-mura, Yazu-gun, Tottori pref. $\left(35^{\circ}\right.$ $20^{\prime} \mathrm{N}$ Lat, $134^{\circ} 7^{\prime} \mathrm{E}$ Long).

N-675. Nashibara 1 Nsb-1-2

From $A_{12}$ horizon, 13 to $25 \mathrm{~cm}$ below surface. 
N-676. Nashibara 2 Nsb-2-1

$3660 \pm 120$

1710 B.c.

From A horizon, 10 to $20 \mathrm{~cm}$ below surface of a lower terrace, $\mathrm{S}$ of site from which $\mathrm{N}-675$ was coll.

III. ARCHAEOLOGIC SAMPLES

Izuhara series

$$
\text { A. Japan }
$$

Charcoal in slag from ancient copper refinery at Izuhara-cho, Shimoagata-gun, Nagasaki pref. (34 $13^{\circ}$ N Lat, $129^{\circ} 13^{\prime}$ E Long). According to documents copper mines in this area were operated during 674 to 927 A.D. and since 1486 A.D. Coll. 1968 and subm. by F. Kamiide, Taishu Mine, Toho Zinc Co.

N-559. Izuhara 1

Modern

From slag deposit $>1.5 \mathrm{~m}$ thick, overlain by $40 \mathrm{~cm}$ surface soil.

N-560. Izuhara 2

From exposed slag deposit $25 \mathrm{~cm}$ thick.

N-561. Izuhara 3

From slag deposit $15 \mathrm{~cm}$ thick, overlain by $30 \mathrm{~cm}$ surface soil.
A.D. 1780

$170 \pm 105$

$400 \pm 120$

A.D. 1550

N-562. Izuhara 4

Modern

From exposed slag deposit.

\section{Aparri series}

\section{B. Philippines}

Piece of wood from sunken ship in $20 \mathrm{ft}$ of water, imbedded in $2 \mathrm{ft}$ sand at coast of Aparri, Luzon I., Philippines $\left(18^{\circ} 20^{\prime} \mathrm{N} \mathrm{Lat}, 121^{\circ}\right.$ $50^{\prime}$ E Long). Coll. and subm. 1969 by H. Ito, Soriamont Development Co.

\section{N-656. Aparri 1}

\section{N-667. Aparri 2}

Both samples are from separate pieces.

\section{Sinde series}

$$
\text { C. Africa }
$$

A.D. 1695

$255 \pm 105$

A.D. 1700

Charcoal from 18 in. below surface at various points of single component Iron age village site, $7 \mathrm{mi}$ from Livingstone, S Province, Zambia (17 $45^{\prime} \mathrm{S}$ Lat, $25^{\circ} 45^{\prime} \mathrm{E}$ Long). Cultural materials excavated indicate immediately post-Kalomo occupation. Coll. 1967 and subm. by J. O. Vogel, Livingstone Mus.

N-563. Sinde 1 (ZLM-23) A.D. 1225 
N-564. Sinde 2 (ZLM-24)

N-565. Sinde 3 (ZLM-25)

Comment (J.O.V.): dates confirm typologic seriation defined by Vogel (1969), in which Sinde material was described as styles of pottery already known from Kalomo-type ceramics as well as a range of types in Tonga Diaspora tradition. Cultural deposit is very shallow suggesting fairly short occupation and carbon determinations suggest median date of late 12th century.

\section{Simonga series}

Burnt wooden poles from single component Iron age village site in Simonga Forest Preserve, $12 \mathrm{mi}$ from Livingstone, S Province, Zambia (17 $46^{\prime} \mathrm{S}$ Lat, $25^{\circ} 43^{\prime} \mathrm{E}$ Long). Cultural materials indicate assoc. with Sinde and Simbusenga sites. Coll. 1967 and subm. by J. O. Vogel.

\section{N-571. Simonga 1 (ZLM-26) \\ N-572. Simonga 2 (ZLM-27) \\ N-573. Simonga 3 (ZLM-28) \\ Comment (J.O.V.): dates confirm typologic seriation based on} Kamangoza and Simbusenga excavations and presence of people assoc. with early Tonga tradition in Victoria Falls region by 12th century.

\section{Mukuni series}

Charcoal from 36 in. below surface, just above interface with underlying sterile Kalahari sands, at village of Mukuni, Livingstone Dist., $S$ Province, Zambia (17 $54^{\prime} \mathrm{S}$ Lat, $25^{\circ} 56^{\prime}$ E Long). Cultural materials excavated with this sample indicate assoc. with Sinde and Simonga series with some small admixture of Kalomo elements (Vogel, 1969). Samples come from areas ca. $50 \mathrm{~m}$ apart. Coll. 1969 and subm. by J. O. Vogel.

N-678. Mukuni 1 (ZLM-29)

N-679. Mukuni 2 (ZLM-30)

\section{A.D. 1195}

$755 \pm 105$

$720 \pm 110$

A.D. 1230

$220 \pm 170$

\section{N-668. Chundu (ZLM-31)}

Modern

Charcoal fragment from 24 in. below surface, Chundu Farm, Livingstone Dist., S Province, Zambia (17 $35^{\prime} \mathrm{S}$ Lat, $25^{\circ} 41^{\prime} \mathrm{E}$ Long), from a sealed pottery vessel assoc. with iron hoe and unidentified seeds. Vessel was Kamangoza Class 2 type (Vogel, 1969) suggesting Early Iron age con- 
text. Coll. 1969 and subm. by J. O. Vogel. Comment: larger error due to shortage of sample.

\section{Mwanamaimpa series}

Charcoal from various depths at Mwanamaimpa Mound, Namwala Dist., S Province, Zambia ( $15^{\circ} 59^{\prime} \mathrm{S}$ Lat, $26^{\circ} 7^{\prime} \mathrm{E}$ Long). Early Iron age levels overlain by later horizons containing pottery of unknown type. Coll. 1968 and subm. by B. M. Fagan, Univ. of California.

N-581. Mwanamaimpa 1 (MM/RC/01) 24 in. below surface. Assoc.: Ila.

N-582. Mwanamaimpa 2 (MM/RC/03) 50 in. below surface. Assoc.: Ila.

N-583. Mwanamaimpa 3 (MM/RC/08) 108 in. below surface. Assoc.: Mid-Iron age.

N-584. Mwanamaimpa 4 (MM/RC/09) 126 in. below surface. Assoc.: Mid-Iron age.

N-585. Mwanamaimpa 5 (MM/RC/16) 192 in. below surface. Assoc.: Early Iron age.

N-586. Mwanamaimpa 6 (MM/RC/17) 197 in. below surface. Assoc.: Early Iron age.

N-578. Mwanamaimpa 7 (MM/RC/19)
A.D. 1215

$735 \pm 105$

$$
605 \pm 105
$$$$
\text { A.D. } 1345
$$

A.D. 1015

$935 \pm 110$

A.D. 1025

$$
925 \pm 110
$$

$$
1370 \pm 130
$$
A.D. 580

$1170 \pm 115$ A.D. 780

$925 \pm 110$

120 in. below surface. Assoc.: Mid-Iron age.

\section{Basanga series}

Charcoal from various depths at Basanga mound, Namwala Dist., $S$ Province, Zambia ( $15^{\circ} 50^{\prime} \mathrm{S}$ Lat, $26^{\circ} 5^{\prime} \mathrm{E}$ Long). Early Iron age levels overlain by later horizons containing pottery of unknown type. Coll. 1968 and subm. by B. M. Fagan.

\section{N-588. Basanga $1 \quad($ BS/RC/01)}

18 in. below surface. Assoc.: Ila.

\section{N-589. Basanga 2 (BS/RC/03)}

24 in. below surface. Assoc.: Ila.

N-590. Basanga 3 (BS/RC/10)

71 in. below surface. Assoc.: Mid-Iron age.
A.D. 1310

$640 \pm 110$

A.D. 1130

$$
820 \pm 110
$$

$$
845 \pm 110
$$

A.D. 1105 
N-591. Basanga $4 \quad(\mathrm{BS} / \mathrm{RC} / \mathrm{15})$

102 in. below surface. Assoc.: Mid-Iron age.

N-592. Basanga 5 (BS/RC/22)

108 in. below surface. Assoc.: Early Iron age.

\section{N-593. Basanga 6 (BS/RC/16)}

139 in. below surface. Assoc.: Early Iron age.

N-594. Basanga $7 \quad(\mathrm{BS} / \mathrm{RC} / 17)$

183 in. below surface. Assoc.: Early Iron age.

\section{N-595. Basanga $8 \quad(\mathrm{BS} / \mathrm{RC} / 18)$}

$865 \pm 110$

A.D. 1085

A.D. 1095

$855 \pm 105$

A.D. 1070

$880 \pm 100$

$1160 \pm 115$ A.D. 790

$1220 \pm 120$

A.D. 730

195 in. below surface. Assoc.: Early Iron age.

General Comment for Mwanamaimpa and Basanga series (B.M.F.): dates indicate Early Iron age occupation of both mounds ended in 8th century A.D.; 11th century date for main occupation of both mounds seems well established. They were probably abandoned long before present inhabitants arrived.

\section{N-493. Nyang'oma Rock Shelter}

$2640 \pm 120$

690 в.C.

Charcoal fragment 20 to $35 \mathrm{~cm}$ below surface in Nyang'oma Rock shelter, Mwanza East area, Tanzania (2० $27^{\prime} \mathrm{S}$ Lat, $33^{\circ} 41^{\prime} \mathrm{E}$ Long), occurring with "Late Stone age" industry characterized by small crescents, assoc. with sherds of "Kansyore Ware." Coll. 1968 and subm. by R. C. Soper, Brit. Inst. Hist. and Archaeol., E Africa.

N-650. Gatare Forest

$$
\begin{array}{r}
1300 \\
\text { A.D. } 650
\end{array}
$$

Charcoal 30 to $36 \mathrm{~cm}$ below surface at Gatare Forest, Mairi Track, Fort Hall Dist., Kenya ( $0^{\circ} 44^{\prime}$ S Lat, $36^{\circ} 47^{\prime}$ E Long), assoc. with obsidian industry of "Late Stone age" type and overlain by pottery-bearing horizon. Coll. and subm. 1969 by R. C. Soper.

\section{Usangi Hospital series}

Samples from site at Usangi Hospital, North Pare Mts., N Tanzania ( $3^{\circ} 42^{\prime} \mathrm{S}$ Lat, $37^{\circ} 39^{\prime} \mathrm{E}$ Long). Area $24 \mathrm{~m}^{2}$ was excavated down to 70 $\mathrm{cm}$ from surface. Finds are homogeneous throughout showing affinities both to Kwale ware and $\mathrm{N}$ Tanzania A-group pottery (Soper, 1967). Suggested archaeologic date: 2nd half of 1st millennium A.D. Coll. 1969 by K. Odner; subm. by H. N. Chittick, Brit. Inst. Hist. and Archaeol., E Africa.

N-646. Usangi Hospital 1

$$
1030 \pm 130
$$

$15 \mathrm{~cm}$ below surface (Sq. 1D).

\section{A.D. 920}


N-647. Usangi Hospital 2

$5180 \pm 135$

$25 \mathrm{~cm}$ bleow surface $(\mathrm{Sq} .6 \mathrm{D})$.

3230 B.c.

\section{N-648. Usangi Hospital 3}

$1430 \pm 270$

Between 40 and $50 \mathrm{~cm}$ below surface (Sq. 2D).

General Comment (K.O.): N-646 and N-648 are consistent with archaeologic dates. Contamination is most likely explanation for early dates of $\mathrm{N}-647$ as material from this level is same as that from which the other charcoal samples were taken.

N-649. Mwanga 3A

A.D. 960

Charcoal between 0 to $10 \mathrm{~cm}$ below surface, at iron smelting site Mwanga 3A in North Pare Mts., N Tanzania (3 $40^{\prime} \mathrm{S}$ Lat, $37^{\circ} 36^{\prime} \mathrm{E}$ Long), assoc. with iron slag, tuyeres, quartz flakes, and parts of vessel similar to NE Tanzania B group dated at Bombo to A.D. $890 \pm 110$ (N-348, Radiocarbon, 1968, v. 10, p. 343). Coll. 1969 by K. Odner; subm. by H. N. Chittick.

\section{N-651. Prospect Farm Stone Bowl site (PF-1)}

$$
2910 \pm 110
$$

Charcoal from Prospect Farm Stone Bowl site, Nakuru Dist., Kenya $\left(0^{\circ} 35^{\prime} \mathrm{S}\right.$ Lat, $36^{\circ} 11^{\prime} \mathrm{E}$ Long), alt. $6700 \mathrm{ft}$, occurring as a concentrated patch, 30 to $50 \mathrm{~cm}$ horizontal extent and $15 \mathrm{~cm}$ depth, within continuous layer of ash, $15 \mathrm{~cm}$ thick and ca. $1 \mathrm{~m}$ below surface, associated with concentration of bones and artifacts including stone bowl, polished stone axe, and deeply incised potsherds. Coll. and subm. 1969 by M. N. Cohen, Brit. Inst. Hist. and Archaeol., E Africa.

\section{N-652. Deloraine Farm site (Del-1)}

$$
1070 \pm 110
$$

Charcoal from Deloraine Farm site, Nakuru Dist., Kenya $\left(0^{\circ} 11^{\prime}\right.$ $S$ Lat, $35^{\circ} 48^{\prime}$ E Long), scattered throughout whole of $10 \mathrm{~cm}$ layer, 60 to $70 \mathrm{~cm}$ below surface, of $2 \times 4 \mathrm{~m}$ test pit, assoc. with heavy concentration of fauna and pottery with incised and punctate decorations otherwise unknown in region. Coll. and subm. 1969 by M. N. Cohen.

\section{Keringet Cave series}

Charcoal from various depths of stone bowl cremation site near Gilgil (Brown, 1966), Nakuru Dist., Kenya (0²1' S Lat, $35^{\circ} 40^{\prime}$ E Long). Coll. and subm. 1969 by M. N. Cohen.

\section{N-653. Keringet Cave 1 (KH-3)}

$$
2910 \pm 115
$$

From depth 65 to $70 \mathrm{~cm}$, scattered in Layer 14 of test pit, outside region of cremation pits. Assoc. with new type of impressed potsherds, presumably predating stone bowl assemblage. 
N-654. Keringet Cave 2 (KH-1)

From 60 to $65 \mathrm{~cm}$ below surface of shelter, occurring as a consolidated chunk, assoc. with cremated remains and artifacts of Njoro River Cave type.

\section{N-655. Keringet Cave 3 (KH-2)}

From depth 50 to $55 \mathrm{~cm}$ within burial pits, assoc. with artifacts of Njoro River Cave type.

\section{REFERENCES}

Aramaki, S., $1965,{ }^{14} \mathrm{C}$ data at Ito pyroclastic flow, Aira Caldera- ${ }^{14} \mathrm{C}$-age of the Quaternary deposits in Japan XXII: Earth Science, v. 80, p. 38.

Brown, J., 1966, The excavation of burial mounds at Ilkek, near Gilgil, Kenya: Azania, v. 1, p. 59-78.

Hsu, Y.-C. et al., 1965, Low background counter for carbon-14 dating: Chinese Jour. Phys., v. 3, p. 1-9.

Isshiki, N. et al., 1965, Radiocarbon Dating: Geol. News, v. 133, p. 20-27.

Kigoshi, Kunihiko and Kobayashi, Hiromi, 1966, Gakushuin natural radiocarbon measurements V: Radiocarbon, v. 8, p. 54-73

MacFarlan, E., 1961, Radiocarbon dating of Late Quaternary deposits in south Louisiana, Geol. Soc. America Bull: v. 72, p. 129-135.

Nakamura, J., 1969, Palynological study of the boring core from Kochi city: Research repts., Kochi Univ., v. 18, Nat. Sci. no. 2.

Omura, A., Konishi, K., and Hamada, T., 1969, Comparison of ${ }^{14} \mathrm{C}$ with ${ }^{230} \mathrm{Th}$ and ${ }^{231} \mathrm{~Pa}$ ages in some hermatypic corals: Fossils, v. 15 , p. 53-65.

Oya, M. 1969, Geomorphology and flooding of the plain in the middle and lower reaches of the Tone River in Kanto Plain: Geog. Jour., v. 78, p. 43-56.

Shepard, F. P., 1964, Sea level changes in the past 6000 years: possible archaeological significance: Science, v. 143 , p. $574-576$.

Soper, R. C., 1967, Kwale: an early Iron Age site in south-eastern Kenya, and Iron Age sites in north-eastern Tanzania: Azania, v. 2, p. 1-36.

Vogel, J. O., 1969, Kamangoza: an introduction to the Iron Age cultures of the Victoria Falls Region: Oxford Univ. Press, Nairobi.

Williams, G. E., 1969, Glacial age of piedmont alluvial deposits in the Adelaide area, South Australia: Australian Jour. Sci., v. 32, p. 257.

Yamasaki, Fumio. Hamada, Tatsuji, and Fujiyama, Chikako, 1966, RIKEN natural radiocarbon measurements II, Radiocarbon, v. 8, p. 324-339.

1968, RIKEN natural radiocarbon measurements IV: Radiocarbon, v. 10 p. $333-345$. 